\title{
Importance of Rectal Urgency in Childhood Irritable Bowel Syndrome
}

TO THE EDITOR: We read with interest the article titled "The incidence of irritable bowel syndrome in children using the Rome III criteria and the effect of trimebutine treatment" by Karabulut et $\mathrm{al}^{1}$ that is a rare and a valuable study from our country.

As the author stated, functional dyspepsia and urgency are very common symptoms in irritable bowel syndrome (IBS) patients. Although the association with the functional dyspepsia is known there is limited study regarding the frequency of rectal urgency in literature and we consider this knowledge to be of importance. In adult studies it was reported that rectal urgency is common especially in diarrheal type of IBS, although it is not included in the Rome III criteria. Cheng et $\mathrm{al}^{2}$ in their study indicated $83 \%$ association between rectal urgency and IBS in only 6 cases. Karabulut et $\mathrm{al}^{1}$ reported frequency of rectal urgency as $66.7 \%$ in children. That shows rectal urgency is an important symptom in childhood IBS and shed light on new studies about its utility as a diagnostic criteria.

It was also shown that colonic motility is related with opioid receptors in colon by pathophysiologic studies. Another important point from the study is the successful result with the trimebutine maleate that is effective on opioid receptors. Current treatments are not effective enough against IBS symptoms and new treatment modalities are necessary. Trimebutin maleate, one of the treatment options, shows encephalin like effect and binds to intestine smooth muscle $\mu$ and $\delta$ receptors. It is stimulatory in case of hypomotility states and inhibitory in case of hypermotility states. ${ }^{3}$ Although the success rate is approximately $50 \%$ in adults, it is important to note that it was more than $90 \%$ in children population in terms of pain and abdominal discomfort. It suggests that opioid receptors have an important role in the pathogenesis of childhood IBS. In a recent study, it was shown that urgency symptoms improved markedly with Eluxadoline that exerts its ef- fects by opioid receptors. ${ }^{4}$ However, recovery of the IBS symptoms was evaluated according to the last week's pain and abdominal discomfort. Therefore, it is yet a question weather the drug has a long-term effect or not.

Last but not least, IBS and related syndromes (chronic pain syndrome, fibromyalgia and chronic pelvic pain syndrome) can affect the quality of life and cause decline in school performance in children. Even though the primary aim of this study is not to evaluate quality of life, successful treatment of pain and abdominal discomfort symptoms with trimebutine maleate will support the healthy psychosocial development and quality of life in children.

Naci Topaloğlu, ${ }^{1}$ Erdem Akbal $^{2}$ and Şule Yıldırım ${ }^{1}$

Departments of ${ }^{1}$ Pediatrics and ${ }^{2}$ Gastroenterology, Medical Faculty, Çanakkale Onsekiz Mart University, Çanakkale, Turkey

1. Karabulut GS, Beşer OF, Erginöz E, Kutlu T, Cokuğraş FÇ, Erkan $\mathrm{T}$. The incidence of irritable bowel syndrome in children using the Rome III criteria and the effect of trimebutine treatment. J Neurogastroenterol Motil 2013;19:90-93.

2. Cheng XF, Tan J, Tan KL. [Clinical analysis of six cases with juvenile primary fibromyalgia syndrome.] Zhonghua $\mathrm{Er} \mathrm{Ke} \mathrm{Za} Z \mathrm{Zhi}$ 2005;43:863-865. [Chinese]

3. Delvaux M, Wingate D. Trimebutine: mechanism of action, effects on gastrointestinal function and clinical results. J Int Med Res 1997; 25:225-246.

4. Dove LS, Lembo A, Randall CW, et al. Eluxadoline benefits patients with irritable bowel syndrome with diarrhea in a phase 2 study. Gastroenterology 2013;145:329-338, e1.

\section{Conflicts of interest: None.}

